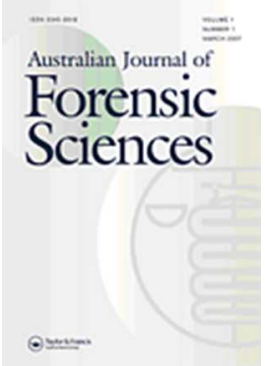

\title{
A comparison of human and pig decomposition rates and odour profiles in an Australian environment
}

\begin{tabular}{|r|l|}
\hline Journal: & Australian Journal of Forensic Sciences \\
\hline Manuscript ID & TAJF-2017-0161.R1 \\
\hline Manuscript Type: & Original Paper \\
\hline Keywords: & $\begin{array}{l}\text { Forensic taphonomy, volatile organic compounds, decomposition, GC } \times \text { GC- } \\
\text { TOFMS, AFTER }\end{array}$ \\
\hline
\end{tabular}

SCHOLARONE $^{\text {Ix }}$
Manuscripts 


\section{Abstract}

4

\section{Keywords:}

\section{A comparison of human and pig decomposition rates and odour profiles in an}

\section{Australian environment}

Cadaver-detection dogs are trained to locate victim remains; however, their training is challenging due to limited access to human remains. Animal analogues, such as pigs, are typically used as alternative training aids. This project aimed to compare the visual decomposition and volatile organic compound (VOC) profile of human and pig remains in an Australian environment, to determine the suitability of pig remains as human odour analogues for cadaver-detection dog training. Four human cadavers and four pig carcasses were placed in an outdoor environment at the Australian Facility for Taphonomic Experimental Research (AFTER) across two seasons. Decomposition was monitored progressively in summer and winter. VOCs were collected onto sorbent tubes and analysed using comprehensive two-dimensional gas chromatography - time-of-flight mass spectrometry. Visual observations highlighted the differences in decomposition rates, with pig remains progressing through all stages of decomposition, and human remains undergoing differential decomposition and mummification. Chemical and statistical analysis highlighted variations in the composition and abundance of VOCs over time between the odour profiles. This study concluded that the visual decomposition and VOC profile of pig and human remains was dissimilar. However, in cooler conditions the results from each species became more comparable, especially during the early stages of decomposition.

Forensic taphonomy; volatile organic compounds; decomposition; GC $\times$ GC-TOFMS; AFTER

*Corresponding Author: Dr Maiken Ueland. Email: maiken.ueland@uts.edu.au 


\section{1. Introduction}

27

28

29

30

31

32

33

34

35

36

37

38

39

40

41

42

43

44

45

46

47

48

49

50

51

52

53

54

55

56

57

58

59

In cases where remains are concealed either naturally (i.e. disaster victims) or intentionally (i.e. homicides), investigators need a reliable search tool to assist in the search and recovery of victim remains. As a body decomposes, the organic components of the body are slowly broken down into smaller gas and liquid molecules ${ }^{1}$. Complex mixtures of volatile organic compounds (VOCs) represent many of the gaseous compounds released as by- and end-products of the decomposition process ${ }^{1}$. VOC mixtures form a dynamic odour profile that insects and canines can both utilise to track and locate remains ${ }^{1,2}$. Due to their superior olfactory systems (compared to humans), canines have an enhanced ability to detect specific odours. For example, cadaver-detection dogs are trained to detect the scent of decomposition in order to assist with victim recovery operations ${ }^{1,2}$. These dogs are trained using a range of natural and artificial training aids to continually imprint them with the variety of decomposition odour profiles that could be encountered in criminal and mass disaster investigations $^{1,2}$.

In many countries, ethical and legal restrictions prevent the use of human cadavers in the training of cadaver-detection dogs and in decomposition research studies generally ${ }^{1}$. This has led handlers and researchers alike to utilise either small samples of human remains (e.g. cadaveric blood, bone, soft tissue, or decomposition fluid) or human analogues ${ }^{1}$. Until 2016 , decomposition studies in Sydney, Australia rarely used human remains, and particularly not outside of a mortuary setting. Studies ${ }^{3,4,5,6,7,8,9,10}$ instead utilised pig carcasses as human analogues. Pigs were considered suitable human decomposition analogues due to the similarity of their internal anatomy and gut biota to humans. They are also more readily available and often do not require ethics approval (i.e. for adults not bred for research purposes $)^{11}$. However, the recently established Australian Facility for Taphonomic Experimental Research (AFTER) is a licensed facility that meets the ethical and legal requirements necessary for research involving human cadavers, but such research can only be conducted in the local environment of Sydney. It is therefore important to compare the process of decomposition between the two species to determine the suitability of pig carcasses as human decomposition analogues. This is also important in understanding the applicability of using human analogues as training aids for cadaver-detection dogs.

While the decomposition process is a complex, variable and multi-faceted process, it is often classified into five stages: fresh, bloat, active decay, advanced decay, and dry remains or skeletonization ${ }^{12,13,14}$. However, the complex mechanisms involved in human decomposition can result in the occurrence of differential decomposition. This refers to the 
60 presence of multiple stages of decomposition occurring concurrently on a body ${ }^{15}$. Factors that

61 can influence differential decomposition include insect activity, differential temperatures, 62 partial concealment of remains in soil or water, sun versus shade, etc. ${ }^{15}$. Due to the difficulty 63 and subjectivity in assessing decomposition through conventional staging, several studies 64 have attempted to provide a more objective method of determination ${ }^{16,17}$. Total Body Score 65 (TBS) is a numeric system for quantifying the amount of decomposition at any given point in 66 time $\mathrm{e}^{3,15,16,18}$. The system works by classifying the remains into regions and then scoring each 67 of these regions individually based on specific criteria ${ }^{3,15,16,18}$. The individual scores are 68 combined to produce a final TBS. This system caters to the presence of differential 69 decomposition across the body by evaluating each region of the body, rather than assigning 70 an arbitrary classification that may not represent the overall state of the remains $s^{3,15,16,18}$. As 71 each stage is characterized by its own set of distinct qualities, each stage is also known to 72 have its own odour signature ${ }^{23}$. It is for this reason that dogs require a variety of training aids, 73 to account for the dynamic nature of decomposition odour across all stages of the process.

This study involved the collection of a headspace sample from above human and pig 75 remains onto sorbent tubes. The samples were analysed using comprehensive two76 dimensional gas chromatography coupled with time-of-flight mass spectrometry $(\mathrm{GC} \times \mathrm{GC}$ 77 TOFMS). Sorbent tubes were used rather than other sample collection methods such as solidphase microextraction (SMPE) fibres due to their suitability for use in field studies and based on a history of similar studies that have utilised this technique with success $8,9,10,19,20,21$. $\mathrm{GC} \times \mathrm{GC}$ provides a greater degree of separation which makes it more useful than one dimensional GC in comprehensive screening studies ${ }^{7}$. TOFMS is more suitable for non-target analyses and has a faster acquisition rate that can accommodate the narrower peaks produced by $\mathrm{GC} \times \mathrm{GC}^{22}$. GC $\times \mathrm{GC}$-TOFMS has been recognised as a successful method for 84 decomposition odour analysis and has become common practice in a number of recent studies $^{7,8,9,21,23,24,25,26}$.

The aim of this study was to compare the decomposition process of human and pig remains in the Sydney environment, both visually and chemically through VOC analysis. A recent $\operatorname{study}^{27}$ found that animal analogues were not accurate representations of human 89 decomposition in a Tennessee environment. Specifically, the study reported a faster rate of 90 decomposition for pig remains and much greater variability in the process of human 91 decomposition. The current study intends to not only compare the rate of decomposition but 92 to also compare the decomposition odour of pig and human remains in the natural Sydney 93 environment and across the entire decomposition process. 


\section{Materials and method}

96

97

98

99

100

101

102

103

104

105

106

107

108

109

110

111

112

113

114

115

116

117

118

119

120

121

122

123

124

125

126

127

\subsection{Experimental Design}

The study was conducted in a natural outdoor Australian environment in Western Sydney on land privately owned by the University of Technology Sydney (UTS). The human remains were located at AFTER while the pig carcasses were placed in the same location but outside the AFTER fence to comply with the licencing requirements of AFTER. The research area consisted of open eucalypt woodland, defined as Cumberland Dry Sclerophyll Forest. Soils at the site are broadly classified as sandy clay loam or gravelly sandy clay, with a $\mathrm{pH}$ range from 5.5-6.5. The site encompasses approximately 4.86 hectares of land surrounded by a high-security fence with closed-circuit television (CCTV) cameras operating continuously.

To compare the human and pig VOC profile, domestic pig carcasses (Sus scrofa domesticus L.) weighing 60 - $80 \mathrm{~kg}$ were compared to donated human cadavers weighing 60 - $90 \mathrm{~kg}$. In order to account for potential seasonal differences, two experimental trials were conducted. The summer trial was conducted from February 2 - March 8, 2016 using two human cadavers (16-02 and 16-03) and two pig carcasses (SP1 and SP2), and the winter trial was conducted from July 27 - August 30, 2016, also using two human cadavers (16-17 and 16-18) and two pig carcasses (WP1 and WP2).

Conforming to the Australian Code of Practice for the Care and Use of Animals for Scientific Purposes (2004), animal ethics approval was not required, as the pig carcasses used in this study did not include living or foetal subjects. The pig carcasses were purchased postmortem from a licensed abattoir. All pigs were killed by captive-head bolt following the standard guidelines for Australian abattoir procedures. All carcasses were wrapped in large polyethylene tarpaulins for transportation to the site. The pig remains were placed directly onto the soil surface approximately $3 \mathrm{~m}$ apart within $1 \mathrm{~h}$ of death. No visible signs of decomposition were observed on the carcasses when they were placed at the site.

The four human cadavers used in this study were acquired through the UTS Body Donation Program overseen by the Surgical and Anatomical Science Facility (SASF) at UTS. Consent was provided by all donors to use their remains for the purposes of research at AFTER, in accordance with the NSW Anatomy Act (1977). The research project was approved under the UTS Human Research Ethics Committee Program Approval (UTS HREC REF NO. ETH15-0029). All donors were placed directly onto the soil surface at AFTER, in the centre of individual $5 \mathrm{~m} \times 5 \mathrm{~m}$ plots. In the summer trial, the plots for donors 16-02 and 
128 16-03 were approximately $10 \mathrm{~m}$ apart, while in the winter trial the plots for donors $16-17$ and 129 16-18 were adjacent to one another, with the remains approximately $3 \mathrm{~m}$ apart. All four 130 donors were male and arrived with no visible signs of decomposition, with the exception of 131 donor 16-17 who demonstrated signs of early decomposition.

For all trials, a control site located a minimum of $3 \mathrm{~m}$ from the remains was established to collect control samples that would identify the background VOCs naturally produced in the surrounding environment. Anti-scavenging cages were placed over the remains when they were not being sampled. These were designed to discourage vertebrate animals from scavenging, while still allowing for invertebrate scavenging to occur and exposure to normal weather conditions.

Visual observations were recorded (i.e. written notes and photographs) once per 139 sampling day. In addition to general observations, the remains were also assigned a TBS adapted from Megyesi et al. ${ }^{16}$. The summer trial was carried out for 34 experimental days and 141 the winter trial for 79 experimental days to cover the range of decomposition processes 142 typically experienced in this environment. Sample collection was performed at varying 143 intervals depending on the expected decomposition rates with additional sampling days in the 144 winter trial $(n=15)$ compared to the summer trial $(n=12)$ due to the slower decomposition 145 rates observed during the early post-mortem period of the winter trial. The stage of 146 decomposition was reported in experimental days (ED).

147 For each trial, a Hobo Weather Station equipped with a Hobo U30 No Remote 148 Communication data logger (OneTemp, Marleston, NSW, Australia) was used to monitor 149 temperature $\left({ }^{\circ} \mathrm{C}\right)$ and rainfall $(\mathrm{mm})$ at an hourly rate.

\subsection{VOC sample collection}

152 The method for sampling VOCs was adapted from headspace VOC collection used in 153 previous research ${ }^{7,8,9,21,23,24,25,26}$. An aluminium hood was placed over the remains and left for $15415 \mathrm{~min}$ to allow the VOCs to accumulate in the headspace. An ACTI-VOC low flow air 155 sampling pump (Markes international Ltd., Llantrisant, UK) was connected to one end of a 156 dual sorbent tube containing Tenax TA and Carbograph 5TD (Markes international Ltd.), 157 with the other end of the tube attached to the sampling port on the aluminium hood. The 158 pump was used to actively draw $1 \mathrm{~L}$ of headspace through the sorbent tube at a flow rate of $159100 \mathrm{~mL} / \mathrm{min}$. All tubes were sealed with brass storage-caps after collection, wrapped in 160 aluminium foil and placed in an airtight glass container for transportation and storage in the 161 laboratory. The sorbent tubes were stored at $4{ }^{\circ} \mathrm{C}$ until the sample analysis was performed. 


\section{$2.3 G C \times G C-T O F M S$ analysis}

To enable peak area normalisation, an internal standard consisting of $2 \mu 1$ of $150 \mathrm{ppm}$ bromobenzene (GC grade, Sigma-Aldrich, Castle Hill, NSW, Australia) in methanol (HPLC Grade, Sigma-Aldrich) was injected onto each sorbent tube prior to analysis.

A Markes Unity 2 Thermal Desorber and Series 2 ULTRA multi-tube autosampler

168

169

170

171

172

173

174

175

176

177

178

179 (Markes International Ltd.) was used to perform thermal desorption of the sorbent tubes. Each sorbent tube was heated to $300{ }^{\circ} \mathrm{C}$ for $4 \mathrm{~min}$ to allow thermal desorption of the compounds before being collected onto a general-purpose cold trap (TenaxTA/Carbograph 1TD) at $-10{ }^{\circ} \mathrm{C}$. The trap was desorbed at $300{ }^{\circ} \mathrm{C}$ for $3 \mathrm{~min}$ with a split flow of $20 \mathrm{~mL} / \mathrm{min}$.

The thermal desorption unit was connected to a Pegasus ${ }^{\circledR} 4 \mathrm{D}$ GC $\times$ GC-TOFMS (LECO, Castle Hill, NSW, Australia) using a $1 \mathrm{~m}$ uncoated silica transfer line (Markes International Ltd.) held at $120^{\circ} \mathrm{C}$ by way of an Ultimate Union Kit (Agilent Technologies, Mulgrave, NSW, Australia). A $30 \mathrm{~m} \times 0.25 \mathrm{~mm}$ inner diameter (ID), $1.40 \mu \mathrm{m}$ film thickness $\mathrm{Rxi}^{\circledR}-624$ Sil MS column (Restek Corporation, Bellefonte, PA, USA) was used as the first dimension column, and a $2 \mathrm{~m} \times 0.25 \mathrm{~mm}$ ID, $0.50 \mu \mathrm{m}$ film thickness Stabilwax ${ }^{\circledR}$ column (Restek Corporation) was used as the second dimension column. The columns were joined with a SilTite $\mu$-Union (SGE Analytical Science). Helium (high purity, BOC, Sydney, NSW, Australia) was used as the carrier gas at a constant flow rate of $1.00 \mathrm{~mL} / \mathrm{min}$. The first dimension oven was set to $35^{\circ} \mathrm{C}$ and held at this temperature for $5 \mathrm{~min}$ before increasing at a rate of $5{ }^{\circ} \mathrm{C} / \mathrm{min}$ to $240{ }^{\circ} \mathrm{C}$ where it was held for a further $5 \mathrm{~min}$. The offset for the modulator was $+5{ }^{\circ} \mathrm{C}$ relative to the GC first dimension oven temperature and the offset for the second dimension column was $+15{ }^{\circ} \mathrm{C}$ relative to the second dimension oven temperature. The modulation period was $5 \mathrm{~s}$ with a $1 \mathrm{~s}$ hot pulse. The transfer line between the second dimension column and the MS was held at $250{ }^{\circ} \mathrm{C}$. An acquisition rate of $100 \mathrm{spectra} / \mathrm{s}$ was used to target a mass acquisition range of $29-450 \mathrm{amu}$. The ion source was held at $200{ }^{\circ} \mathrm{C}$, the electron ionisation energy was $70 \mathrm{eV}$, and the detector voltage was programmed with a $200 \mathrm{~V}$ offset above the optimized detector voltage determined.

\subsection{Data processing}

ChromaTOF $^{\circledR}$ (version 4.51.6.0; LECO) was used for data processing. A 150 signalto-noise $(S / N)$ ratio was used with a baseline offset of 0.8 . The peak widths for the first and second dimensions were $30 \mathrm{~s}$ and $0.15 \mathrm{~s}$, respectively. The National Institute of Standards and Technology (NIST) Mass Spectral Library was used to establish a list of compounds with a 
196

197

198

199

200

201

202

203

204

205

206

207

208

209

210

211

212

213

214

215

216

217

218

219

220

221

222

223

224

225

226

227

228

mass spectral match threshold of $80 \%$. Peak alignment was performed between samples using a mass spectral match threshold of $60 \%$ by utilising the Statistical Compare software feature within ChromaTOF $^{\circledR}$. Once aligned, analyte peak areas were normalised based on the peak area of the internal standard. The samples were organised into two classes per analysis: experimental ( $n=24$ for the summer trial and $n=30$ for the winter trial), and control ( $n=12$ for the summer trial and $n=15$ for the winter trial). This procedure was carried out separately for the human and pig samples; however, an additional analysis that combined the two datasets was also performed (i.e. pig vs. human). The additional analysis also consisted of two classes: experimental ( $\mathrm{n}=48$ for the summer trial and $\mathrm{n}=60$ for the winter trial), and control ( $n=24$ for the summer trial and $n=30$ for the winter trial). During alignment, analytes were only retained if found in at least 3 of the total samples for that trial or in $10 \%$ of the samples within a class.

The Statistical Compare software feature was used to calculate the Fisher ratio of each compound detected. A Fisher ratio threshold was established based on a critical $\mathrm{F}$ value $\left(F_{\text {crit }}\right)$ that was calculated using the number of classes, the degrees of freedom per class, and a significance level of 0.05 . Compounds that had a Fisher ratio lower than the $F_{\text {crit }}$ value were excluded. Compounds that arose due to chromatographic artefacts or were a result of column/sorbent bleed were also removed. The final peak table was further processed using Microsoft Excel.

Unscrambler ${ }^{\circledR} \mathrm{X}$ (version 10.5; CAMO Software, Oslo, Norway) was used to perform principal component analysis (PCA). Mean centering, variance scaling and unit vector normalisation were all applied to the datasets prior to PCA. The data was shown to contain no outliers by way of Hotelling's T2 95\% confidence limit.

\section{Results and discussion}

\subsection{Weather Conditions}

During the summer trial, the average daily temperature was $23.5^{\circ} \mathrm{C}$ with a total range of $14.7{ }^{\circ} \mathrm{C}-40.6{ }^{\circ} \mathrm{C}$. A total of $3 \mathrm{~mm}$ of rainfall was recorded during this period. In the winter trial, the average daily temperature was $11.1^{\circ} \mathrm{C}$ with a total range of $1.1{ }^{\circ} \mathrm{C}-27.3^{\circ} \mathrm{C}$. A total of $34.2 \mathrm{~mm}$ of rainfall was recorded during this period.

\subsection{Visual Comparison}

\subsubsection{Summer Trial}


On ED 0 of the summer trial, both human cadavers were defined as being in the fresh stage and the posterior of the torso for donor 16-02 continued to be scored as fresh until ED 16. Both donors developed mild bloat on ED 1, before entering full bloat on ED 6. Donor 1603 developed mild bloat on ED 6 and entered full bloat on ED 10. Bloating began to subside on ED 14 for donor 16-02 and ED 16 for donor 16-03; with both sets of remains displaying post-bloat deflation by ED 21. Active decay was only observed internally and along the soil line/posterior aspect of the torso and upper limbs. In both donors, active decay started between ED $14-21$. Both sets of remains began to transition into advanced decay in these regions as the trial ended from ED $28-35$.

There was a clear trend towards differential decomposition and preservation exhibited by both sets of human remains. Desiccation occurred as early as ED 10 in donor 16-03. The head and neck region and the anterior aspect of both donors were desiccated by ED 14. The posterior aspect of the arms was the only area exhibiting skeletonization in donor 16-02, occurring around ED 21. Donor 16-03 showed the same skeletonization pattern, although the donor had additional skeletonization of the face due to heavy entomological scavenging around the nose. Donor 16-03 first showed signs of skeletonization of the face on ED 28, with skeletonization of the arms developing on ED 35.

The decomposition process was faster for the pig remains compared to the human remains, similar to the study reported in Tennessee ${ }^{27}$. On ED 0 of the summer trial, both carcasses were defined as being in the fresh stage. By ED 1, both carcasses had entered the bloat stage. Bloating had subsided by ED 6 and active decay was observed from ED $6-8$.

250 The progression of active decay was rapid causing skeletonization to occur in the head and 251 limbs for both sets of remains during this time. The carcasses transitioned into advanced 252 decay from ED 10 to ED 35, but also exhibited some desiccation during active decay (ED 6 253 8). This was likely due to the high temperatures exhibited during the summer trial. 254 Desiccation persisted through advanced decay, however, the carcasses showed a more typical 255 trend towards skeletonization, which began during active decay and increased over time until the end of the trial (ED $6-35)$.

Despite the use of anti-scavenging cages, some animal scavenging occurred as a result

258 of burrowing underneath the cages. The presence of animal scavenging during this trial 259 meant that some of the remaining desiccated tissue was removed manually, with both 260 carcasses becoming fully skeletonised around ED 28 - 35. The warmer daily temperatures 261 and minimal rainfall during the summer trial were likely responsible for the faster 262 decomposition rates and desiccation observed compared to the winter trial. Differences 
263 between the pig and human decomposition during this trial was likely due to biological

264 dissimilarities between the two species such as distribution of body weight rather than 265

266

267

268

269

270

271

272

273

274

275

276

277

278 environmental variables, since the remains were placed in the same location. Additionally, the regions accessed and degree of insect activity varied between species demonstrating greater soft tissue loss and skeletonization in pig remains.

\subsubsection{Winter Trial}

In the winter trial, human and pig remains were classified as being in the fresh stage of decomposition on ED 0. However, donor 16-17 had visibly undergone some decomposition and was defined as being in the late stages of fresh decomposition, with green discolouration and fluid blisters indicating that enzymatic decay processes had already commenced. Donor 16-18 maintained some areas with fresh tissue throughout the entire trial, with the feet and lower legs experiencing minimal decomposition. Bloat only occurred on one of the two human remains. Donor 16-17 displayed mild bloating on ED 27, though this had subsided by ED 34. Similar to the summer trial, active decay was confined internally and was only visually apparent in the head and neck regions. Active decay was observed during ED 13 - 34 for donor 16-17 and ED 16 - 34 for donor 16-18. The remains in the winter trial also demonstrated a trend towards differential decomposition and preservation. Desiccation was first observed on donor 16-18 on ED 16 and on donor 16-17 on ED 20. In both cases, the desiccation began in the head and neck region, followed by the arms, and progressed slowly across the anterior of the body until ED 79, marked by a slow continual darkening of the desiccated skin. Skeletonization was first observed on ED 16 of donor 16-18 around the lower jaw, due to entomological activity in this area. On ED 41, the upper torso of 16-17 began to show signs of skeletonization. By ED 50 this had extended to also include the posterior aspect of the arms and head on both 16-17 and 16-18, and the groin of 16-17 on ED 79.

The pig remains also progressed slower through the decomposition timeline in comparison to their summer trial counterparts. WP1 showed signs of bloating on ED 9, and full bloat from ED 13 - 27. WP2 showed signs of bloating on ED 6, and full bloat from ED 9 - 27. Both carcasses exhibited post-bloat deflation as they progressed into active decay on ED 34, fully deflating by ED 50. Unlike the human remains, the pig remains exhibited far less desiccation during the trial period. The skin of WP1 became darker and leathery on ED 64; however, it had not fully desiccated by ED 79. The pigs showed a greater precedence towards soft tissue loss and therefore skeletonization. Skeletonization of the head and neck 
297 began on ED 41 in both carcasses, with the limbs also showing signs of skeletonization on

298 ED 50. The areas of skeletonization increased and became more prominent until the end of 299 the trial on ED 79. The cooler daily temperatures and increased prevalence of rainfall during 300 the winter trial were likely responsible for the slower decomposition rates observed during 301 the winter trial, and these findings were comparable to other studies in the same region using 302 pig remains ${ }^{9,10}$.

303 In both trials, the human remains demonstrated differential decomposition with the 304 posterior progressing into active decay but the anterior becoming mummified. In contrast, the 305 pig remains progressed through the more typical stages of decomposition resulting in 306 skeletonization. These differences can be predominantly associated with the degree of 307 entomological activity and regions accessed in the bodies. Insect activity was heavily 308 localised to the head and groin for human remains during the earlier postmortem period 309 allowing time for soft tissue to become mummified elsewhere on the remains. Ultimately, the 310 anterior mummified tissue acted as a protective shell for the entomological activity which 311 eventually led to active decay of the posterior in contact with the soil. In contrast, 312 entomological activity although initiated in the head and groin of the pig remains, was 313 observed across the entire remains leading to more rapid soft tissue loss and exposure of 314 bone. This may be due to the different structure of the pig remains whereby the lack of a 315 defined neck and shortened limbs provides a more uniform body mass for the insect larvae to 316 move across and consume.

317

318 3.2.3 Total Body Scoring

319 Total body scoring was used to semi-quantify the degree of decomposition over time 320 for all sets of remains. During the summer trial, TBS ranged from $3-32$, while in the winter 321 trial TBS ranged from 3 - 27 (Figure 1). Overall, the TBS of remains during winter were 322 lower than those recorded in summer which correlates with visual observations. In summer, 323 the pig remains scores were generally higher than those recorded for the human remains, with 324 this difference increasing over time. In contrast, the TBS recorded in winter were comparable 325 for human and pig remains throughout the trial. These trends indicate that decomposition 326 rates vary greatly in warmer temperatures between human and pig remains; with the 327 decomposition rates becoming more comparable for both species in cooler temperatures. This 328 is likely because decomposition occurs at a slower rate in cooler temperatures and when rain 329 is more prevalent ${ }^{9,10}$. These results also correlate with the study in Tennessee ${ }^{27}$ reporting a 330 faster rate of decomposition for pig remains. However, when insect activity was absent (as it 
331 was for the first 100 days of the Tennessee study and for ED 0 - 34 of this study at AFTER 332 during winter), the rate of pig and human decomposition was more comparable ${ }^{27}$. While 333 providing some valuable insight, the original criteria and scoring system outlined by Megyesi 334 et al. ${ }^{16}$ was unsuitable for scoring decomposition of human remains in a temperate Sydney environment and a revised system is currently being developed.

336

\subsection{Chemical VOC Comparison}

The Statistical Compare software feature and Fisher ratio filtering was used to filter out compounds based on their individual $\mathrm{F}_{\text {crit }}$ values from the experimental classes (human and pig remains). The filtering process selects the compounds that are statistically relevant based on their Fisher ratio (i.e. the ratio of between-class variance to within-class variance) being higher than the $\mathrm{F}_{\text {crit }}$ (4.05 [Summer Trial] and 4.01 [Winter Trial]), meaning the compound is specific to a particular class of samples (i.e. experimental) ${ }^{31-35}$. Background VOCs from the environment were manually removed, as were VOCs known to originate from the experimental process, such as solvents and aerosols used at the field site. In the summer trial, a total of 77 VOCs were deemed statistically significant in differentiating the experimental from the control samples, with 74 of these believed to result from the decomposition process based on previous literature ${ }^{1,4-10,20,21,23-26}$. Seventy (70) of these VOCs were detected in the human decomposition odour profile, while all 74 were detected in the pig decomposition odour profile. Hence, there were no VOCs unique to human remains in the summer trial. In the winter trial, a total of 29 VOCs were deemed statistically significant in differentiating the experimental from the control samples, with 28 of these believed to result from the decomposition process based on previous literature ${ }^{1,4-10,20,21,23-26}$. Twenty-three (23) of these VOCs were detected in the human decomposition odour profile, while all 28 were detected in the pig decomposition odour profile. Hence, there were no VOCs unique to human remains in the winter trial.

An average abundance was calculated for each VOC detected from human remains and pig remains in summer and winter. These compounds were sorted into one or more of the following classes: alcohols, aldehydes, aromatics, carboxylic acids, esters, ethers, hydrocarbons, ketones, nitrogen-containing, sulfur-containing, and other (compounds not within these classes). The total class abundance (the sum of the abundances of all VOCs in a class) for each class was calculated and presented graphically in Figures 2 and 3.

In the summer trial (Figure 2), the most abundant compound class detected in human remains were hydrocarbons, followed by alcohols and aromatics. These classes were also 
365 detected in similar odour studies using human remains conducted by Vass ${ }^{25,36}$ and Cablk ${ }^{26}$ 366 who both reported hydrocarbons, alcohols and aromatics in their respective studies. For the 367 pig remains in the summer trial, carboxylic acids were the most abundant class detected, 368 followed by aromatics and hydrocarbons. Carboxylic acids, aromatics and hydrocarbons were 369 also detected in several pig studies conducted by Perrault ${ }^{4-7,10,20}$ and Forbes ${ }^{8,9,28}$ in the same 370 environment as the current study. In the winter trial (Figure 3), the most abundant class 371 detected in human remains were esters, followed by hydrocarbons and aldehydes. Like those 372 detected in the summer trial, the classes detected in the winter trial were also detected in 373 similar odour studies using human remains conducted by $\operatorname{Vass}^{25,36}$ and $\mathrm{Cablk}^{26}$ who both 374 reported esters and aldehydes in addition to hydrocarbons. Hydrocarbons were the most 375 abundant class detected for pig remains in the winter trial, followed by sulfur-containing 376 compounds and esters. These classes were also detected in several pig studies conducted by 377 Perrault ${ }^{4-7,10,20}$ and Forbes ${ }^{8,9,28}$ in the same environment as the current study. Pig remains also 378 demonstrated a higher abundance in the few halogenated compounds (in the 'other' class) 379 that were detected during the winter trial. However this was rarely seen in other studies.

380 These results indicate that the two profiles share many of the same compounds; 381 however, the composition and abundance of these compounds is rarely similar at any given 382 point in time during the decomposition process. It was only during the early stages of the 383 trials when human and pig decomposition rates were more comparable that the two odour 384 profiles shared similarities in the composition and abundance of their odour profiles. While 385 the specific compounds present play an important role in the overall odour profile, the ratio 386 and abundance of the VOCs at any given point in time is also integral to this profile, 387 particularly for training cadaver-detection dogs. This accounts for one of the major chemical 388 differences between the decomposition odour profiles of pig and human remains.

389 PCA was performed to determine the statistical variation of human and pig 390 decomposition odour profiles. The 74 compounds in the summer trial and 28 compounds in 391 the winter trial that were determined to be of significance were used in the analyses. For the 392 summer trial (Figure 4a), the first principal component (PC-1) accounted for the highest 393 amount of variation (24\%) in the dataset followed by the second principal component (PC-2), 394 which explained $15 \%$ of the variance in the dataset. Samples showed intra-variability within 395 an individual class which likely correlates with differences in the decomposition process 396 between the two human donors as a result of factors such as their body mass, genetics, diet, 397 and age. The samples also showed inter-variability due to differences in the rate of 398 decomposition between human and pig remains. Samples collected on Day 16, 21, 28 and 35 
399 when the human remains demonstrated an advanced decomposition stage formed a close 400 cluster indicating minimal variation in the odour profile due to reduced amounts of soft tissue 401 remaining. The Day 16, 21, 28 and 35 samples from both species are separated across both 402 principal components showing a variation in the VOCs released from the individual samples 403 of the pig and human remains.

404 For the winter trials (Figure 4b), PC-1 explains $29 \%$ of the variance while PC-2 405 explains $17 \%$ of the variance in the dataset. Despite the decomposition being more similar 406 during the winter trial, there exists a notable spread of samples across both principal 407 components. Intra-day variation in the VOC profiles of human and pig remains resulted in 408 spread amongst the samples collected on the same day. This can be correlated with the 409 difference in the visual comparison recorded during the field experimental trials.

410 The extent of statistical variation between the human and pig odour profiles suggests 411 that, although the compounds detected using pig remains accounts for those detected using 412 human remains, the ratio and abundance of these compounds over time demonstrates 413 dissimilar odour signatures. This is likely due to the variation in decomposition rates 414 observed whereby soft tissue loss was rapid in the pig remains resulting in skeletonization, 415 but differential decomposition was observed in the human remains with mummified tissue 416 forming on the anterior. Since there is little known about which aspects of the odour profile 417 are utilized by the cadaver-detection dogs to recognise their target, the significance of this 418 dissimilarity is not fully understood and needs to be further investigated involving cadaver419 detection dog trials ${ }^{2,29,30}$. Based on the extent of analysis that was possible in this study, it is 420 recommended that human remains be used as training aids where available. In regions where 421 legal and ethical restrictions prevent the use of human cadaveric materials, the use of pig 422 remains as training aids should be conducted with caution until an understanding of the 423 significance of the difference in odour profiles between pig and human remains is achieved. 424 Such information is important to further our understanding of the science of canine olfaction, 425 and is particularly important for handlers who may be challenged in court on their testimony 426 regarding training protocols of cadaver-detection dogs.

427

\section{4. Conclusion}

The visual findings of this study suggest that pig carcasses are not reliable analogues 430 for describing human decomposition patterns after early decomposition in temperate Sydney 431 environments. However, this cannot be confirmed based on the low number of replicates and 
432 further replicate analyses are currently being performed to ascertain if these trends are 433 repeatable. To date, the results of this study support recent findings from Tennessee ${ }^{27}$ that 434 reported a visual difference between the decomposition of human cadavers and animal 435 analogues (namely pig and rabbit). If confirmed with future studies, these findings will have 436 a significant impact on the use of pig remains to understand the process of human 437 decomposition in the Sydney environment, and particularly their use in estimating time since 438 death.

439 A comparison of the chemical odour profiles found that while the pig remains may 440 account for the VOCs detected from human remains, the variation in ratio and abundance 441 yield dissimilar odour profiles. This finding was supported by PCA analyses which 442 demonstrated statistical variation between the human and pig decomposition odour profiles 443 during both seasonal trials. The comparison of seasonal conditions for this study identified 444 that during the cooler months, human and pig decomposition became more comparable than 445 during the warmer months. This was also reflected in the VOC profiles, with the samples 446 collected in winter being more comparable than those collected in summer. Until further 447 replication is carried out to produce confirmatory findings, the results of this study suggest 448 that cadaver-detection dogs in Sydney, Australia should continue to be trained on the odour 449 of human remains, rather than pig remains to ensure enhanced capabilities when deployed in 450 the field. 


\section{References}

1. Iqbal MA, Nizio KD, Ueland M, Forbes SL. Forensic decomposition odour profiling: A review of experimental designs and analytical techniques. Trends in Analytical Chemistry. 2017;91:112-124.

2. Riezzo I, Neri M, Rendine M, Bellifemina A, Cantatore S, Fiore C, Turillazzi E. Cadaver dogs: Unscientific myth or reliable biological devices? Forensic Science International. 2014;244:213-221.

3. Marhoff SJ, Fahey P, Forbes SL, Green H. Estimating post-mortem interval using accumulated degree-days and a degree of decomposition index in Australia: a validation study. Australian Journal of Forensic Sciences. 2015;48(1):1-13.

4. Perrault KA, Stefanuto PH, Stuart BH, Rai T, Focant JF, Forbes SL (2015). Detection of decomposition volatile organic compounds in soil following removal of remains from a surface decomposition site. Forensic Science, Medicine, and Pathology. 2015;11(3):376-387.

5. Perrault KA (2015). Novel odour analysis of soils associated with decomposed remains. University of Technology Sydney. Unpublished thesis

6. Perrault KA, Stefanuto PH, Stuart H, Rai T, Focant J-F, Forbes SL (2015). Reducing variation in decomposition odour profiling using comprehensive two-dimensional gas chromatography. Journal of Separation Science. 2015;38:73-80.

7. Perrault KA, Nizio KD, Forbes SL. A comparison between one-dimensional and twodimensional gas chromatography for decomposition odour profiling using inter-year replicates field trials. Chromatographia. 2015;78(15):1057-1070.

8. Forbes SL, Perrault KA. Decomposition odour profiling in the air and soil surrounding vertebrate carrion. PLoS ONE. 2014;9(4):1-12.

9. Forbes S, Perrault K, Stefanuto PH, Nizio K, Focant JF. Comparison of the decomposition VOC profile winter and summer in a moist, mid-latitude $(\mathrm{Cfb})$ climate. PLoS ONE. 2014;9(11):1-11.

10. Perrault K, Rai T, Stuart B, Forbes SL. Seasonal comparison of carrion volatiles in decomposition soil using comprehensive two-dimensional gas chromatography - time of flight mass spectrometry. Analytical Methods. 2015;7:690-698.

11. Stokes KL, Forbes SL, Tibbett M. Human versus animal: Contrasting decomposition dynamics of mammalian analogues in experimental taphonomy. Journal of Forensic Sciences. 2013;58(3):583-591. 
487

488

489

490

491

492

493

494

495

496

497

498

499

500

501

502

503

504

505

506

507

508

509

510

511

512

513

514

515

516

517

518

519

520

12. Payne J. A summer carrion study of the baby pig Sus Scrofa Linnaeus. Ecology. 1965;46(5):592-602.

13. Janaway RC, Percival SL, Wilson AS. Decomposition of human remains. In: Percival SL. Microbiology and aging. New York, NY, USA: Springer Science + Business Media, LLC; 2009.

14. Dolinak D, Matshes E, Lew EO. Postmortem changes. In: Forensic pathology:

Principles and practice. Burlington, MA, USA: Academic Press; 2005.

15. Troutman L, Moffatt C, Simmons T. A preliminary examination of differential decomposition patterns in mass graves. Journal of Forensic Sciences. 2014;59(3):621626.

16. Megyesi MS, Nawrocki SP, Haskell NH. Using accumulated degree-days to estimate the postmortem Interval from decomposed human remains. Journal of Forensic Sciences. 2005;50(3):1-9.

17. Vass AA. The elusive universal post-mortem interval formula. Forensic Science International. 2011;204:34-40.

18. Moffat C, Simmons T, Lynch-Aird J. An improved equation for TBS and ADD: Establishing a reliable postmortem interval framework for casework and experimental studies. Journal of Forensic Sciences. 2016;16(S1):S201-S207.

19. Markes International - thermal desorption and TOF MS [Internet]. Llantrisant (UK): Markes International Ltd; [date of publication unknown] [cited 2017 Aug 3]. Available from: https://www.markes.com/.

20. Perrault K A, Stuart B H, Forbes S L. A longitudinal study of decomposition odour in soil using sorbent tubes and solid phase microextraction. Chromatography. 2014;1(3):120-140.

21. Dekeirsschieter J, Stefanuto PH, Brasseur C, Haubruge E, Focant JF. Enhanced characterization of the smell of death by comprehensive two-dimensional gas chromatography - time of flight mass spectrometry (GCXGC-TOFMS). PLoS ONE. 2012;7(6):1-16.

22. Cotter R. Time-of-flight mass-spectrometry for the structural-analysis of biological molecules. Analytical Chemistry 1992;64(21):A1027-A1039.

23. Stadler S, Stefanuto PH, Brokl M, Forbes SL, Focant JF. Characterization of volatile organic compounds from human analogue decomposition using thermal desorption coupled to comprehensive two-dimensional gas chromatography-time-of-flight mass spectrometry. Analytical Chemistry. 2013;85:998-1005. 
521 24. Hoffman E, Curran A, Dulgerian N, Stockham R, Eckenrode B. Characterization of 522 the volatile organic compounds present in the headspace of decomposing human remains. Forensic Science International. 2009;186:6-13.

524 25. Vass A, Smith R, Thompson C, Burnett M, Dulgerian N, Eckenrode B. Odor analysis 525 of decomposing buried human remains. Journal of Forensic Science. 2008;53(2):384$526 \quad 391$

527 26. Cablk M, Szelagowski E, Sagebiel J. Characterization of the volatile organic compounds present in the headspace of decomposing animal remains, and compared with human remains. Forensic Science International. 2012;220:118-125.

27. Alapo L. Humans-Pigs-Rabbits study to impact court cases worldwide. Knoxville (US): The University of Tennessee; 2016. [cited 2017 Aug 3]. Available from: http://ntoday.utk.edu/2016/04/27/humanspigsrabbits-decomposition-study-impactcourt-cases-worldwide/

28. Forbes S, Troobnikoff A, Ueland M, Nizio K, Perrault K. Profiling the decomposition odour at the grave surface before and after probing. Forensic Science International. 2016;259:193-199

29. Tipple C, Caldwell P, Kile B, Beussman D, Rushing B, Mitchell N, Whitchurch C, Grime M, Stockham R, Eckenrode B. Comprehensive characterization of commercially available canine training aids. Forensic Science International. 2014;242:242-254. human remains detection canines utilizing a non-contact, dynamic airflow volatile concentration technique. Forensic Science International. 2011;217(1-3):32-38. Focant, Exploring new dimensions in cadaveric decomposition odour analysis, Anal. Methods 2015;7:2287-2294. Available from: http://dx.doi.org/10.1039/C5AY00371G 32. A.C. Beckstrom, E.M. Humston, L.R. Snyder, R.E. Synovec, S.E. Juul, Application of comprehensive two-dimensional gas chromatography with time-of-flight mass spectrometry method to identify potential biomarkers of perinatal asphyxia in a nonhuman primate model, J. Chromatogr. A 2011;1218:1899-1906. Available from: http://dx.doi.org/10.1016/j.chroma.2011.01.086 .

33. M. Brokl, L. Bishop, C.G. Wright, C. Liu, K. McAdam, J.-F. Focant, Multivariate analysis of mainstream tobacco smoke particulate phase by headspace solidphase micro extraction coupled with comprehensive two-dimensional gas chromatography- 
555 time-of-flight mass spectrometry, J. Chromatogr. A 2014;1370:216-229. Available 556 from: http://dx.doi.org/10.1016/j.chroma.2014.10.057.

557 34. K.D. Nizio, K.A. Perrault, A.N. Troobnikoff, M. Ueland, S. Shoma, J.R. Iredell, P.G. 558 Middleton, S.L. Forbes, In vitro volatile organic compound profiling using $\mathrm{GC} \times \mathrm{GC}$ 559 TOFMS to differentiate bacteria associated with lung infections: a proof-of-concept 560 study, J. Breath Res. 2016;10:26008. Available from: http://dx.doi.org/10.1088/1752$561 \quad \underline{7155 / 10 / 2 / 026008}$.

562 35. P.-H. Stefanuto, K.A. Perrault, S. Stadler, R. Pesesse, H.N. LeBlanc, S.L. Forbes, J.-F. 563 Focant, GC $\times$ GC-TOFMS and supervised multivariate approaches to study human 564 cadaveric decomposition olfactive signatures, Anal. Bioanal.

565 36. Vass A. Odor mortis. Forensic Science International. 2012;222:234-241. 566 
567 Figure 1: Scatter plot of total body scores for all human and pig remains studied.

568

569 Figure 2: Total abundance of compound classes in the summer trial for average human (H)

570 and pig $(P)$ decomposition odour profiles.

571

572 Figure 3: Total abundance of compound classes in the winter trial for average human (H)

573 and pig $(P)$ decomposition odour profiles.

574

575 Figure 4: a) Principal component analysis for the summer trial, the circle shows clustering of

576 the VOC profiles from the human donors on days 16, 21, 28 and 35. b) Principal component

577 analysis for the winter trial. Note: a zoomed in region of the data points in the circle was

578 added for easy viewing.

579

580 


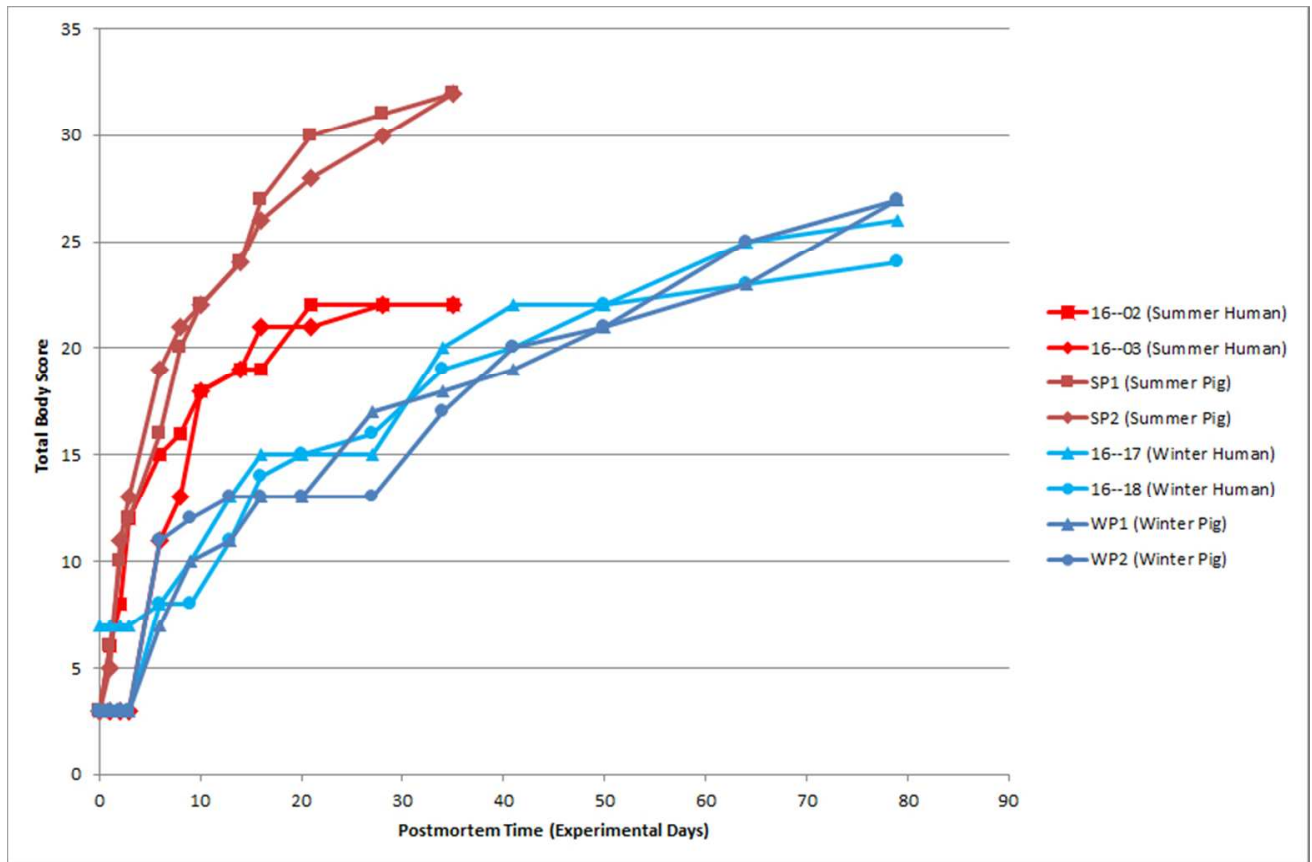

Figure 1: Scatter plot of total body scores for all human and pig remains studied.

\section{$234 \times 155 \mathrm{~mm}(96 \times 96 \mathrm{DPI})$}




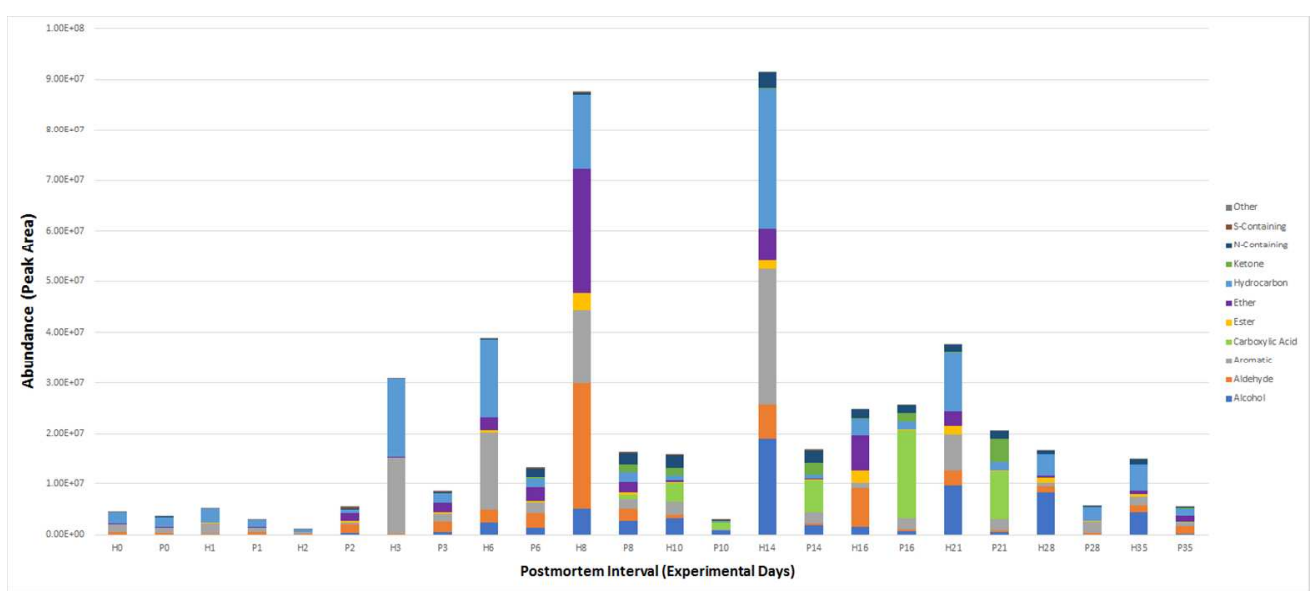

Figure 2: Total abundance of compound classes in the summer trial for average human $(\mathrm{H})$ and pig $(\mathrm{P})$ decomposition odour profiles.

$404 \times 179 \mathrm{~mm}(96 \times 96 \mathrm{DPI})$ 


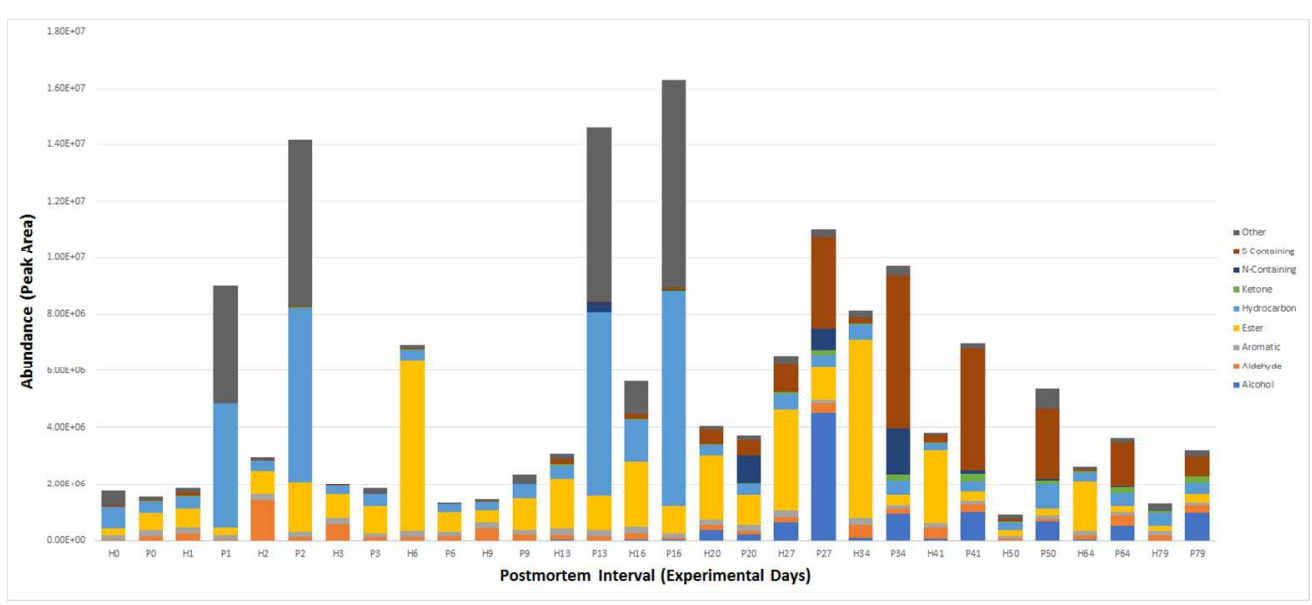

Figure 3: Total abundance of compound classes in the winter trial for average human $(H)$ and pig $(P)$ decomposition odour profiles.

$404 \times 180 \mathrm{~mm}(96 \times 96 \mathrm{DPI})$ 
a.

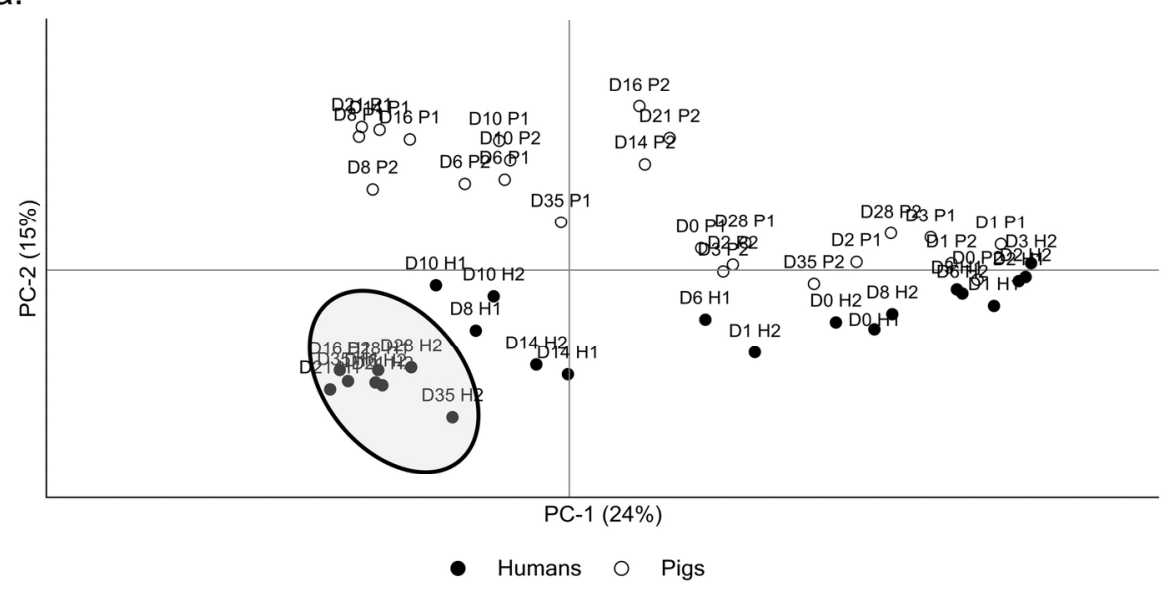

b.

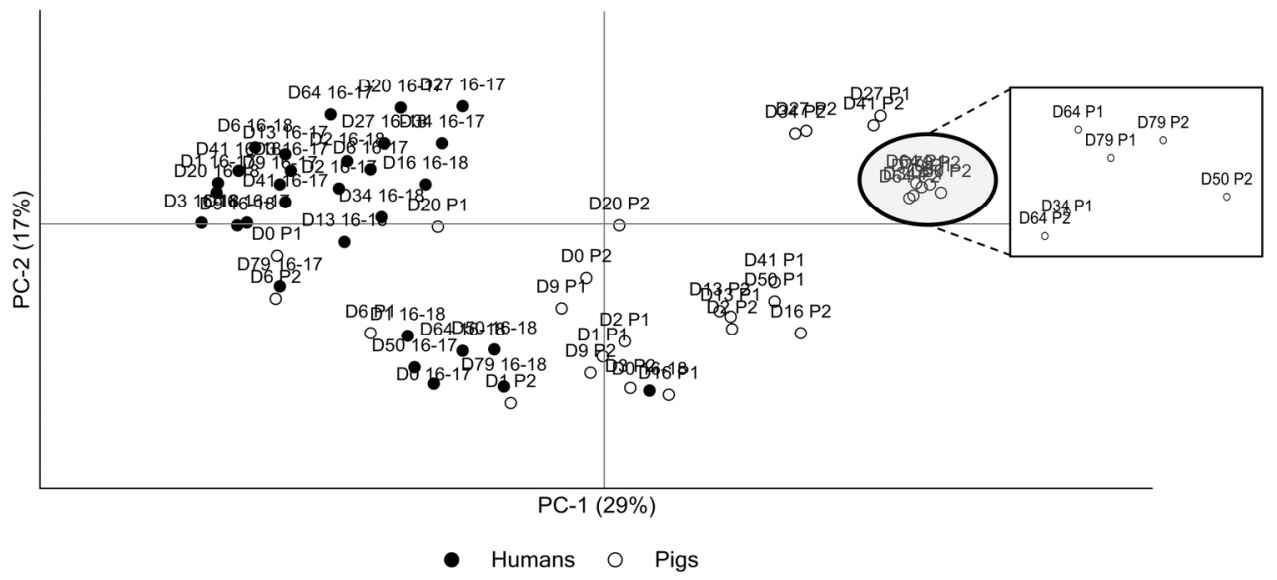

Figure 4: a) Principal component analysis for the summer trial, the circle shows clustering of the VOC profiles from the human donors on days 16, 21, 28 and 35. b) Principal component analysis for the winter trial. Note: a zoomed in region of the data points in the circle was added for easy viewing.

$167 \times 164 \mathrm{~mm}(300 \times 300 \mathrm{DPI})$ 